\title{
Manejo de fístulas faringocutáneas en pacientes operados de faringolaringectomía y reconstrucción inmediata por carcinoma de hipofaringe avanzado
}

\author{
Management of pharyngocutaneous fistulas with microvascular flaps \\ in patients undergoing pharyngeal laryngectomy and immediate \\ reconstruction for advanced hypopharyngeal carcinoma \\ Dr. Miguel de la Parra-Márquez,* Dr. Hugo de León-Camacho,* Dr. Maynard Trejo-Meyer ${ }^{\ddagger}$
}

\footnotetext{
Palabras clave: Hipofaringe, carcinoma, reconstrucción, colgajo microvascular.

Keywords: Hypopharynx, carcinoma, reconstruction, microvascular flap.
}

\section{RESUMEN}

El cáncer de faringe es una neoplasia agresiva y el de hipofaringe es el de peor pronóstico de todos los carcinomas de células escamosas de cabeza y cuello. En los últimos 10 años el autor ha operado 12 pacientes de laringofaringectomía radical y ha realizado reconstrucción inmediata con colgajos microvasculares fasciocutáneos en la UMAE No. 21 del Instituto Mexicano del Seguro Social en Monterrey, Nuevo León, México. Cuatro pacientes (33.3\%) presentaron fístulas salivales, de los cuales, dos cedieron al manejo conservador con sonda nasogástrica durante tres semanas; los otros dos pacientes no cedieron al manejo conservador por presentar trayectos cortos y anteriores, próximos al estoma laríngeo. Las fístulas salivales son una complicación frecuente en las reconstrucciones faríngeas con colgajos microvasculares. Si no hay cierre con manejo conservador durante cuatro semanas, se debe considerar la opción de otro colgajo microvascular para el cierre. El colgajo anterolateral de muslo es una excelente opción en pacientes delgados. Se puede obtener un colgajo con poca grasa que no obstruya el estoma traqueal. Asimismo, el colgajo perforante de la arteria circunfleja superficial es ideal en pacientes con panículo graso en los muslos, donde el colgajo anterolateral cerca del estoma traqueal es muy grueso para la reconstrucción.

\section{ABSTRACT}

Pharyngeal cancer is an aggressive neoplasm, and hypopharyngeal cancer has the worst prognosis of all squamous cell carcinomas of the head and neck. In the last 10 years, the author operated on 12 patients for radical laryngopharyngectomy and performed immediate reconstruction with fasciocutaneous microvascular flaps, at UMAE No. 21 of the Mexican Institute of Social Security in Monterrey, Nuevo León, Mexico. Four patients (33.3\%) had salivary fistulas, of which, 2 yielded to conservative management with a nasogastric tube for three weeks; the other two patients did not yield to conservative handling because they had short and anterior paths, close to the laryngeal stoma. Salivary fistulas are a frequent complication in pharyngeal reconstructions with microvascular flaps. If there is no closure with conservative management for four weeks, the option of another microvascular flap for closure should be considered. The anterolateral thigh flap is an excellent option in lean patients 'low-fat flap can be obtained and does not obstruct the tracheal stoma. Likewise, the superficial circumflex artery perforator flap is ideal in patients with a fat pad on the thighs, where the anterolateral flap near the tracheal stoma is too thick for reconstruction.
* Cirujano plástico.

* Residente de cirugía general, Hospital

Christus Muguerza.

Departamento de Cirugía Plástica y Reconstructiva, UMAE No. 21 del Instituto Mexicano del Seguro Social.

Recibido: 08 noviembre 2020 Aceptado: 19 abril 2021

\section{INTRODUCCIÓN}

$\amalg_{\text {como una complicación frecuente de la }}$ cirugía laringoesofágica con una incidencia reportada entre 3 y $65 \%$, la que además de asociarse con un aumento en la morbilidad y mortalidad, también se asocia con un incremento

Citar como: De la Parra-Márquez M, De León-Camacho H, Trejo-Meyer M. Manejo de fístulas faringocutáneas en pacientes operados de faringolaringectomía y reconstrucción inmediata por carcinoma de hipofaringe avanzado. Cir Plast. 2021; 31 (2): 62-67. https://dx.doi.org/10.35366/102747 
importante en la estancia intrahospitalaria así como en los costos generados. ${ }^{1}$ Entre los efectos secundarios de esta complicación destacan las infecciones, aspiraciones con consecuente compromiso pulmonar, exposición de la arteria carótida y la evolución tórpida de las heridas quirúrgicas. ${ }^{2-9}$

El tratamiento de las fístulas faringocutáneas consiste en un manejo integral que incluye un manejo conservador con antibioticoterapia y ayuno, el cuidado integral de las heridas con desbridamientos, uso de terapia de presión negativa y finalmente el cierre con colgajos. ${ }^{1,10,11}$

Existen múltiples opciones de colgajos utilizables para el cierre de la fístula; sin embargo, cada uno de ellos tiene ciertas desventajas. Algunos pueden utilizarse únicamente para defectos pequeños; el deltopectoral, por ejemplo, presenta una morbilidad importante del sitio donador, que por lo regular necesita un segundo procedimiento quirúrgico. Los colgajos regionales tienden a ser abultados, dificultan su ensamble y sacrifican tejido muscular. Por su parte, los colgajos libres se pueden utilizar para defectos grandes y son maleables; no obstante, se caracterizan por una técnica quirúrgica compleja y un tiempo quirúrgico más prolongado en pacientes que tienden a tener comorbilidades importantes. ${ }^{10}$

La decisión del cierre con colgajos se sugiere después del manejo conservador no exitoso por al menos cuatro semanas, basándose en parámetros clínicos como el tamaño de la fístula y la calidad de los tejidos blandos cervicales. La colocación de tubos salivares durante la reparación de las fístulas reduce la estancia hospitalaria y la severidad de las complicaciones en caso de recurrencia de la fístula. ${ }^{11,12}$ Clásicamente se han utilizado los colgajos regionales, entre los que destacan el pectoral mayor, el deltopectoral y el dorsal mayor así como los colgajos libres, principalmente el radial, el de yeyuno y el anterolateral de muslo. ${ }^{10,13-15}$

\section{MATERIAL Y MÉTODO}

En los últimos 10 años en la UMAE No. 21 del Instituto Mexicano del Seguro Social (IMSS) en Monterrey NL, México se han operado 12 pacientes de faringolaringectomía radical y reconstrucción inmediata con colgajos microvasculares fasciocutáneos plegados en forma tubular para comunicación entre orofaringe y esófago proximal.

Se realizó un análisis descriptivo calculando medidas de tendencia central y dispersión para las variables cuantitativas y análisis de frecuencias para las cualitativas. Se utilizó el programa estadístico SPSS 10.0 (IBM Corp., Chicago IL).

\section{RESULTADOS}

Del total de pacientes, ocho fueron del sexo masculino y cuatro del femenino. La edad promedio $\pm \mathrm{DE}$ fue de $57 \pm 11.44$ años, con rango de 37 a 70 años. La localización más frecuente fue hipofaringe en 10 pacientes $y$ epifaringe en dos.

El colgajo de reconstrucción faríngea más frecuente fue el anterolateral de muslo (ALT), con ocho pacientes $(66.6 \%)$ y el radial con cuatro $(33.33 \%)$. En cuatro pacientes $(33 \%)$

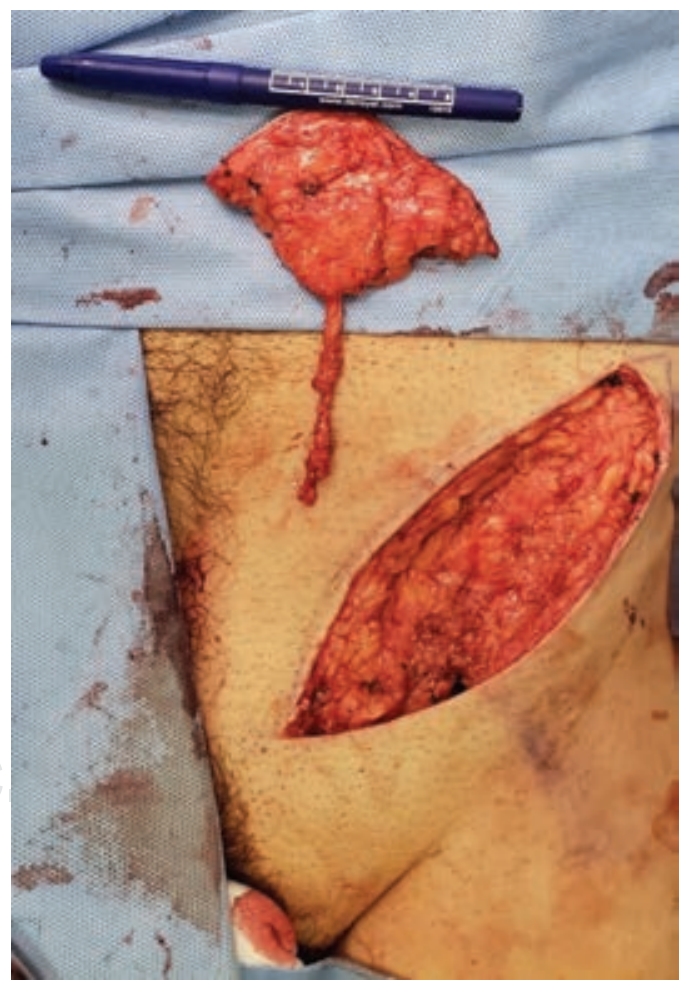

Figura 1: Caso 1. Hombre de 45 años, toma de colgajo basado en la arteria circunfleja iliaca superficial. 


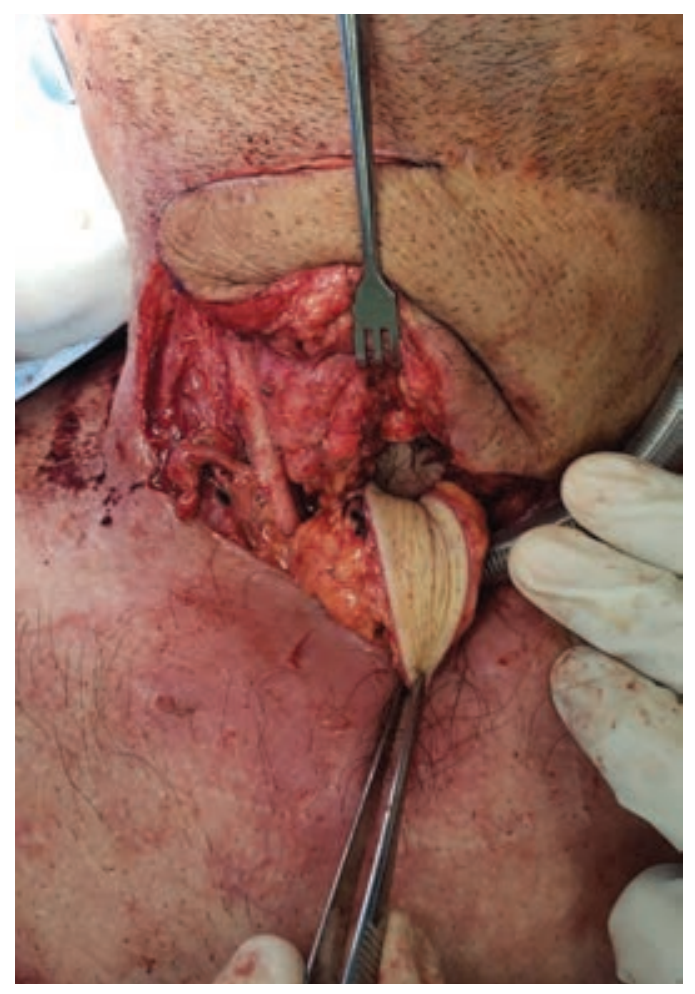

Figura 2: Caso 1. Colocación del colgajo en su sitio receptor. Se observa la arteria carótida interna disecada para la anastomosis vascular. La porción de la isla de piel formará la cobertura interna del área de la fístula faríngea.

se realizaron dos colgajos simultáneos para reconstrucción de faringe y cobertura cutánea: dos pacientes ALT doble, un paciente ALT + pectoral y un paciente ALT + supraclavicular. Todos los colgajos: 12 para faringe y cuatro para cobertura cutánea, fueron viables.

Una paciente reconstruida con colgajo radial presentó estenosis que requirió dilataciones subsecuentes.

Del total de pacientes, cuatro (33.3\%) presentaron fístula salival, de los cuales, dos (50\%) cedieron al manejo conservador con sonda nasogástrica por tres semanas. Los otros dos pacientes (50\%) no cedieron al manejo conservador por mostrar trayectos cortos y anteriores, cercanos al estoma laríngeo. En ambos pacientes el orificio de la fistula fue mayor de $1.5 \mathrm{~cm}$ de diámetro.

Presentamos los dos casos que tuvieron fistula faríngea compleja:

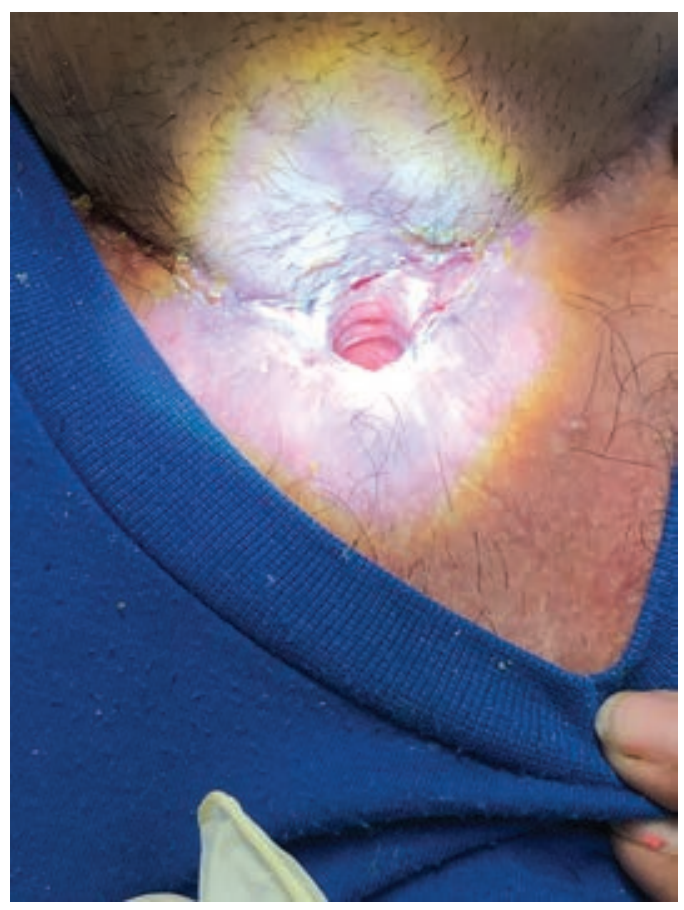

Figura 3: Caso 1. Estoma traqueal tres meses después. Se observa integración total del injerto de piel al área cruenta del colgajo perforante de la arteria circunfleja superficial.

\section{Caso 1}

Hombre de 45 años, con carcinoma recurrente con invasión a hipofaringe. Se realizó faringolaringectomía radical con resección amplia de piel de cuello, reconstrucción con dos colgajos anterolateral de muslo (ALT): uno anastomosado a vasos faciales derechos para reconstrucción de faringe, suturado en forma tubular y anastomosado a la porción superior del colgajo a orofaringe y la porción distal se anastomosó al esófago proximal. Asimismo, el otro colgajo se utilizó para cobertura externa y se anastomosó a vasos faciales izquierdos. La evolución fue satisfactoria retirando la sonda nasogástrica a las tres semanas de la cirugía. Toleró la dieta de líquidos y sólidos y acudió nuevamente tres meses después de la cirugía inicial, con salida de saliva y líquidos por fístula hipofaringeocutánea a nivel de la unión del colgajo ALT y el estoma traqueal en su parte anterior. Se observó orificio de $2 \mathrm{~cm}$ de diámetro con salida abundante de saliva y se optó por un colgajo perforante de 
la arteria circunfleja superficial (SCIP) por ser un colgajo muy delgado, el cual se anastomosó término-lateral a la arteria carótida interna y vena yugular interna. La isla cutánea se suturó a todo el defecto circular, resaltando que en la porción inferior se unió a la porción posterior del estoma traqueal. Se utilizó la parte de la piel para cobertura interna y se colocó injerto de espesor parcial en la parte externa que corresponde al orificio traqueal (Figuras 1 a 3). La evolución fue satisfactoria, tolerando dieta sólida a los seis meses de seguimiento.

\section{Caso 2}

Mujer de 65 años, con carcinoma recurrente de faringe que requirió faringolaringectomía radical. Reconstruida en un inicio con colgajo ALT y posteriormente fístula salival de ocho meses de evolución. Se realizó colgajo nuevo ALT con doble isla cutánea para cobertura interna y externa con anastomosis término-terminal a vasos tiroideos superiores. La isla cutánea para cobertura interna se suturó a todo el defecto

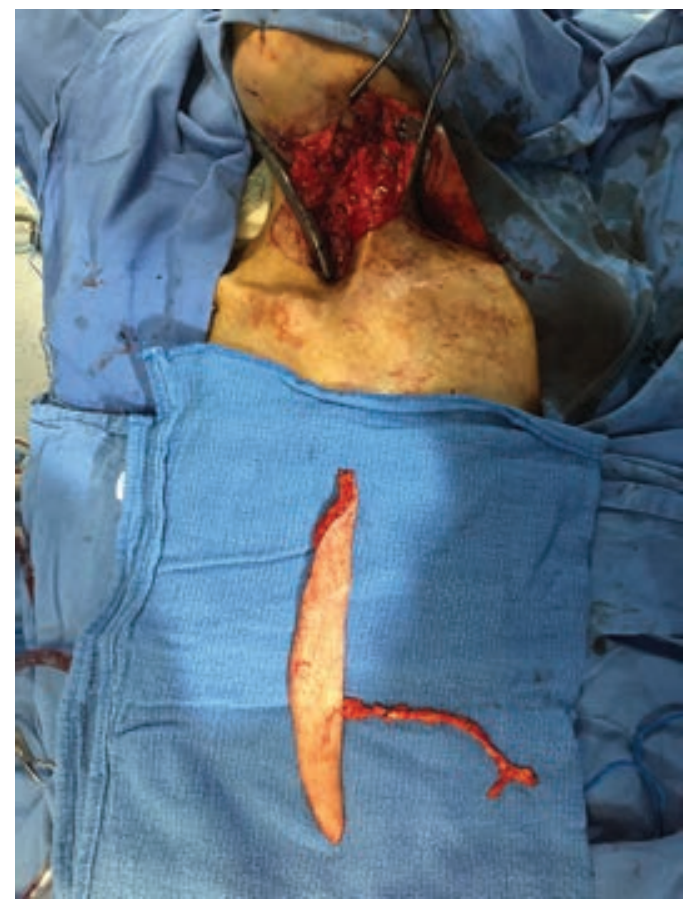

Figura 4: Caso 2. Mujer de 65 años: colgajo anterolateral de muslo delgado. Se observa la disección entre el estoma traqueal y la faringe reconstruida previamente. faríngeo; el borde inferior de la isla de piel de cobertura externa se suturó a la porción libre del estoma traqueal y al defecto cutáneo de la piel del cuello. La evolución fue satisfactoria, tolerando sólidos a seis meses de seguimiento (Figuras 4 y 5).

\section{DISCUSIÓN}

Los pacientes sometidos a laringofaringectomía, ya sea por cáncer de laringe o faringe, pueden reconstruirse de manera satisfactoria con colgajos libres o pediculados.

Los colgajos libres son en la actualidad la primera opción en reconstrucción faríngea, siendo el anterolateral de muslo el que con más frecuencia se utiliza por su gran versatilidad y baja morbilidad en el área donadora. En nuestra serie, ocho pacientes (66\%) fueron reconstruidos de esta manera. ${ }^{13-16}$

La gran versatilidad del colgajo ALT permite realizarlo en forma tubular, éste se conecta en la porción superior a la orofaringe residual y en la inferior al esófago proximal. Las fístulas

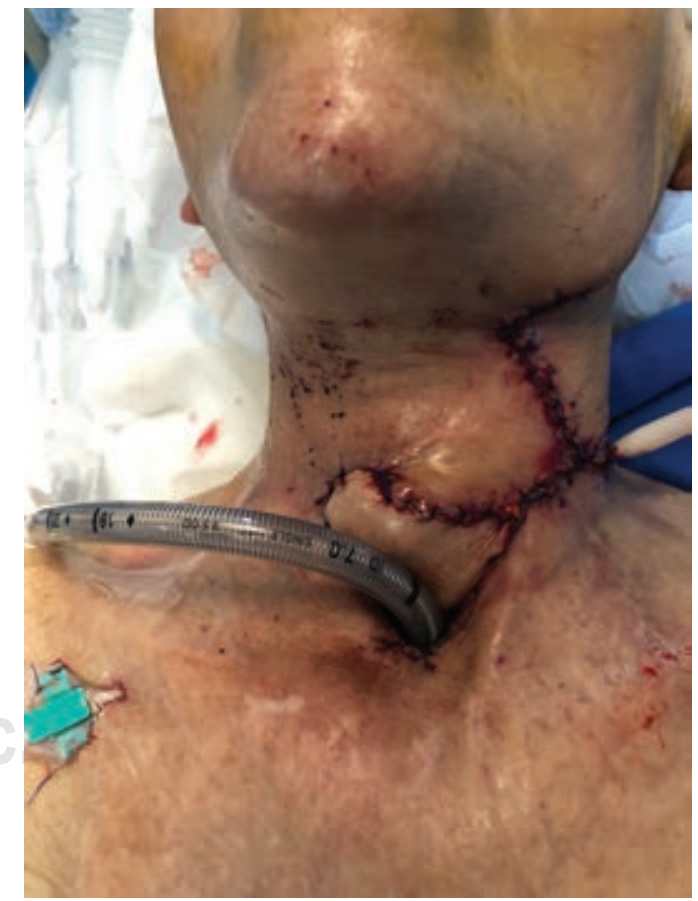

Figura 5: Caso 2. Resultado postoperatorio inmediato: se observa la isla cutánea externa para cobertura de piel del cuello y estoma traqueal. 
faringocutáneas se presentan como una complicación frecuente de la cirugía laringoesofágica, con una incidencia reportada entre 3 y $65 \%$, muy similar a la obtenida en nuestro estudio (33.3\%). 1,10,11 Esta complicación aumenta considerablemente el tiempo de recuperación en los pacientes operados de reconstrucción esofágica así como la incomodidad del paciente debido a la fuga de saliva y a la incapacidad para la ingesta de alimentos por vía oral.

Para su mayor comprensión y manejo, las fístulas cervicales postesofagectomía se han clasificado en cuatro grupos con base en su presentación clínica, datos radiológicos (esofagograma/TAC), hallazgos en reoperaciones y la exploración endoscópica. La fístula tipo I consiste en una fuga subclínica, el tipo II muestra síntomas a nivel cervical, el tipo III se asocia con manifestaciones clínicas respiratorias secundarias a una colección pleural o mediastínica y el tipo IV con un cuadro sistémico secundario a una necrosis de la plastia. ${ }^{1}$ En nuestro estudio, las fístulas presentadas eran tipo II.

La mayor parte de las fístulas faríngeas se manejan de manera conservadora con un tiempo promedio de cierre de cuatro semanas, dependiendo del diámetro del defecto. ${ }^{12}$ En fístulas complejas, el manejo conservador no es efectivo, por lo que es necesario algún tipo de colgajo para su cobertura.

En nuestra serie, cuatro pacientes (33.3\%) presentaron fístula posterior a la reconstrucción faríngea, dos de ellos se manejaron de manera conservadora con cierre de ésta en menos de tres semanas y en los otros dos (50\%) no se obtuvo mejoría con el manejo conservador. En estos últimos dos pacientes se realizó cobertura tanto interna (mucosa faríngea) como externa (piel del cuello). En el primer caso se optó por realizar un colgajo basado en la circunfleja iliaca superficial por ser un colgajo que se puede adelgazar en forma considerable, ya que la fístula era muy baja a nivel del estoma traqueal, donde un colgajo más grueso (en este caso un ALT) podría causar obstrucción del estoma con dificultad para respirar.

En el resultado a largo plazo se observa la integridad del estoma traqueal (Figura 3).

En el caso 2 se optó por un colgajo ALT, ya que la paciente era en extremo delgada, por lo que el ALT con doble isla cutánea logró cubrir tanto en forma interna como externa sin provocar obstrucción del estoma traqueal.

\section{CONCLUSIONES}

Las fístulas salivales son una complicación frecuente en las reconstrucciones faríngeas con colgajos microvasculares (33.3\%). En caso de no presentar cierre con el manejo conservador por cuatro semanas es indispensable tomar en cuenta la opción de otro colgajo microvascular para su cierre. El colgajo ALT es una excelente opción en pacientes delgados, de quienes se puede obtener un colgajo con poca grasa que no obstruya el estoma traqueal. Asimismo, como segunda opción, el colgajo SCIP es el ideal en pacientes con más panículo adiposo en muslos donde el colgajo ALT es demasiado grueso para la reconstrucción cerca del estoma traqueal.

\section{REFERENCIAS}

1. Larburu ES, Gonzales RJ, Elorza OJL et al. Fístula cervical postesofagectomía: diagnóstico y tratamiento. Cir Esp 2013; 91 (1): 31-37.

2. Volkenstein S, Willers J, Noack V, Dazert S, Minovi A. Gesundheitsbezogene lebensqualitat nach behandlung von plattenepithelkarzinomen des oropharynx. Laryngorhinootologie 2015; 94 (8): 509-515.

3. Mehanna H, Evans M, Beasley M, Chatterjee S, Dilkes M, Homer J et al. Oropharyngeal cancer: United Kingdom National Multidisciplinary Guidelines. J Laryngol Otol 2016; 130 (S2): S90-96.

4. Luque SG, Fuentes EM, Sánchez PM, San Pedro SA, Ruiz LRM, Pulgar BD. Resultados funcionales de pacientes tratados con faringolaringectomía y reconstrucción faríngea con colgajo pediculado. Rev Cir 2019; 71 (2): 145-151.

5. Simo R, Rovira A, Townley W. Salvage treatment options after failed primary treatment of hypopharyngeal cancer. Adv Otorhinolaryngol 2019; 83: 135-147.

6. Pracy P, Loughran S, Good J, Parmar S, Goranova R. Hypopharyngeal cancer: United Kingdom National Multidisciplinary Guidelines. J Laryngol Otol 2016; 130 (S2): S104-110.

7. Tirado L, Granados M. Epidemiología y etiología del cáncer de cabeza y cuello. Cancerología 2007; 2: 9-17.

8. Mahalingam S, Spielmann P. Quality of life outcomes following treatment of hypopharyngeal cancer. $A d v$ Otorhinolaryngol 2019; 83: 126-134.

9. Bhide SA, Gulliford S, Kazi R et al. Correlation between dose to the pharyngeal constrictors and patient quality of life and late dysphagia following chemo-IMRT for head and neck cancer. Radiother Oncol 2009; 93 (3): 539-544.

10. Teixeira S, Costa J, Monteiro D et al. Pharyngocutaneous and tracheoesophageal fistula 
closure using supraclavicular artery island flap. Eur Arch Otorhinolaryngol 2018; 275 (7): 1921-1926.

11. Janis JE. Essentials of plastic surgery. 2nd ed. [St. Louis, Mo.]: Quality Medical Publishing, Inc.; Boca Raton, FL: CRC Press/Taylor \& Francis Group. 2014.

12. Morujo RG, Pascual PM, Tucciarone M, Fernández MF, Encabo RS, Guirado TM. Salvage total laryngectomy: is a flap necessary? Braz J Otorhinolaryngol 2020; 86 (2): 228-236.

13. Masià J, Vives L. Colgajo anterolateral del muslo: anatomía quirúrgica, técnica de disección y aplicaciones clínicas. Cir Plast Iberolatinoam 2006; 32 (4): 269-280.

14. Priego R, Trejo D, Haddad JL et al. Colgajo lateral de muslo: aplicaciones clínicas. Cir Plast 2012; 22 (3): 126-133.

15. Reynaldo GMA, Trinchet SR, Pérez FJ et al. Comportamiento de las fístulas faringocutáneas. Hospital Vladimir llich Lenin. Rev Cuba Cir 2010; 49 (2). Disponible en: <http://scielo.sld. cu/scielo.php?script $=$ sci_arttext $\&$ pid $=$ S0034$74932010000200002 \& \operatorname{lng}=\mathrm{es} \& n r m=i s o>$. ISSN 0034-7493

16. Sumarroca A, Rodríguez-Bauzà E, Lop-Gros J et al. Repair of post-laryngectomy pharyngocutaneous fistulas using a pectoralis major flap. Braz J Otorhinolaryngol 2019; 85 (3): 351-356.

Conflicto de intereses: Los autores declaran no tener conflicto de intereses.

Correspondencia:

\section{Dr. Miguel de la Parra-Márquez}

Miguel Hidalgo Núm. 2480, Cons. 212,

Col. Obispado, 64060,

Monterrey, N.L., México.

E-mail: drdelaparra@yahoo.com.mx 